\title{
Measurement and control of high suspended sediment concentration during the Kurobe River sediment flushing with submersible type SMDP
}

\author{
Tetsuya Sumi ${ }^{1,}$, Saichiro Morita ${ }^{2}$, and Hideaki Komiya ${ }^{2}$ \\ ${ }^{1}$ Disaster Prevention Research Institute, Kyoto University, 6110011 Gokasho, Uji, Japan \\ ${ }^{2}$ SMD Engineering, Co., Ltd., Japan
}

\begin{abstract}
In the Kurobe River, coordinated sediment flushing and sediment sluicing of Dashidaira and Unazuki dams have been executed since 2001. From the view point of the comprehensive sediment management in the sediment routing system, monitoring of quantity and quality of sediment transport during these events in rivers and reservoirs is very important. This paper shows the results of continuous measurement of high suspended sediment concentration during sediment flushing and sluicing operation of Unazuki dam in 2015 and 2016. These data have been obtained by submergible type of Suspended Sediment Concentration Measuring System with Differential Pressure Transmitter (hereinafter we call 'submergible type SMDP'), which is designed to be placed underwater. Measured data have good correlation with manual sampling and effectively utilized to control gate operation by real-time basis during the most critical timing producing extreme high concentration just before free flow starts for sediment flushing.
\end{abstract}

\section{Introduction}

In Japan, comprehensive sediment management in the sediment routing system has been implemented in the Kurobe River and the Tenryu River. Sediment management requires measuring the amounts of bed load, suspended load and wash load in a river. Conventional techniques such as manual bottle sampling to measure turbidity and suspended sediment (SS) concentration have been conducted to obtain transport rate of wash load and suspended load $[1,2]$. However, manual sampling has various problems, including noncontinuous and burdensome measurements with respect to time and costs before obtaining results.

Felix et al. recently developed a method to measure high SS concentration of intake water using a vibration tube in order to manage abrasion damages on a hydraulic turbine in a hydraulic power plant [3]. We set up a hypothesis that a change in density can be used to measure the SS concentration in river water, and developed SMDP (Suspended Sediment Concentration Measuring System with Differential Pressure Transmitter) equipment. SMDP continuously and automatically measures the SS concentration as a change in

*Corresponding author: sumi.tetsuya.2s@kyoto-u.ac.jp 
pressure. We have achieved satisfactory results using this SMDP equipment installed in downstream of the Kurobe River [4-8] and the Miwa Dam in the Tenryu river basin.

There are two types of SMDPs which are underwater and water circulation ones. In the former, the entire measurement system is installed directly in water, while in the latter, river water is taken in by a pump and guided through a pipe to a water tank (a differential pressure sensor is installed inside). In 2015, an underwater-type SMDP was installed downstream of the Unazuki Dam in the Kurobe River (Fig. 1). High SS concentrations during sediment flushing were successfully measured in real time. In 2016, we attempted to employ the SS concentration value measured by SMDP to operate the sediment-flushing gate of the dam. Coordinated sediment flushing at the Unazuki Dam requires effective discharge of the suspended load while simultaneously controlling the SS concentration. Consequently, real-time measurements of the SS concentration and its control downstream of the dam by gate operations are important. In this paper, we overview SMDP measurements and the control trials of the sediment flushing concentration from a dam utilizing SMDP measurements.

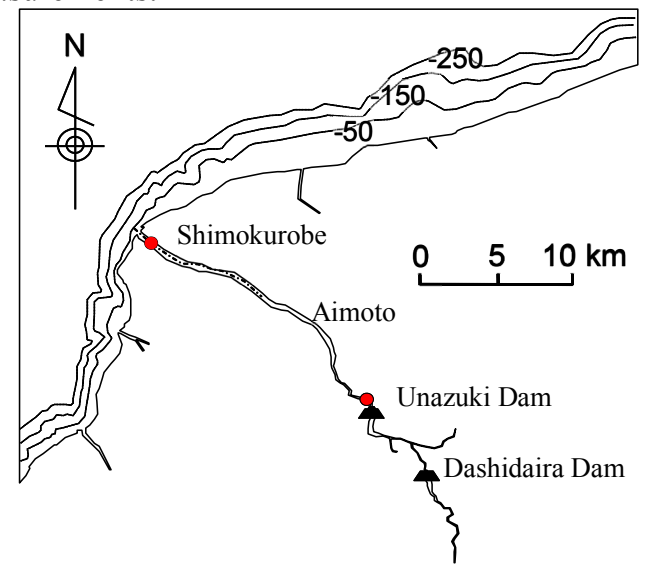

Fig. 1 SMDP measurement points downstream of the Kurobe River

\section{Measurement method}

\subsection{Measurement principles of SMDP}

SMDP continuously monitors high SS concentrations in a river or reservoir during flooding by directly measuring the density of fluid with a highly accurate differential pressure sensor. In running water, the density of water with SS differs from that without SS. If two fixed reference points with vertical spacing $H$ in a fluid are provided, density measurements can be carried out by detecting difference of these pressures $\left(P_{H}\right.$ : high pressure side, $P_{L}$ : low pressure side).

From the Bernoulli's theorem, Eq. (1) is derived where average density, gravitational acceleration, velocity at the high pressure and low pressure points are $\rho, g, V_{H}$ and $V_{L}$, respectively.

$$
\frac{P_{H}}{\rho g}+\frac{V_{H}^{2}}{2 g}=\frac{P_{L}}{\rho g}+H+\frac{V_{L}^{2}}{2 g}
$$

From Eq. (1), the pressure difference $\Delta P$ is calculated by Eq. (2).

$$
\Delta P=P_{H}-P_{L}=\rho g H+\frac{\rho\left(V_{L}^{2}-V_{H}^{2}\right)}{2}
$$


If $V_{H}=V_{L}$ is assumed, $\Delta P$ is calculated by Eq. (3).

$$
\Delta P=P_{H}-P_{L}=\rho g H
$$

Since $g$ and $H$ are known, the average density $\rho$ and the suspended sediment concentration (SSC) can be determined by obtaining the pressure difference.

Here, we can estimate the actual pressure difference to be obtained. Assuming that the water density of SSC $=0 \mathrm{mg} / \mathrm{l}$ is $\rho_{0}\left(\mathrm{~kg} / \mathrm{m}^{3}\right)$, a change in the pressure difference from SSC $=0 \mathrm{mg} / \mathrm{l}$ (I) to $\mathrm{SSC}=10,000 \mathrm{mg} / \mathrm{l}$ (II) can be calculated by Eq. (4) .

$$
\Delta P_{\mathrm{I}}-\Delta P_{\mathrm{II}}=\left(\left(\rho_{0}+0.01\right)-\rho_{0}\right) g H
$$

Assuming $H=1,000 \mathrm{~mm}$, a small pressure change of about $10 \mathrm{mmH}_{2} \mathrm{O}$ must be detectable. To achieve such an accurate measurement, SMDP uses a silicon vibration-type differential pressure sensor with high accuracy and stability $[4,5]$.

\subsection{Underwater-type SMDP installed downstream of the Unazuki Dam}

Fig. 2 (left) shows the underwater-type SMDP installed downstream of the Unazuki Dam. A differential pressure transducer (SS sensor) was installed at the center in an outer cylinder, which had a 500-mm diameter and 700-mm height. The sensor transmitted the pressure difference between the high-pressure side and the low-pressure side in 500-mm intervals in the vertical direction with a narrow tube filled with an enclosed liquid. The SMDP was installed using a concrete jetty on the right bank side about 500-m downstream of the Unazuki Dam. To avoid direct impacts of driftwood and gravel in the discharged water, a storing basket with the built-in SMDP was attached to the downstream side of the jetty using a suspension device (Fig. 2, right).

The central SMDP body was installed so that it was $1,500-\mathrm{mm}$ high from the riverbed (Fig. 2 , center). The measurement span of SMDP was $100,000 \mathrm{mg} / \mathrm{l}$. For example, when the concentration of river water changed from $0 \mathrm{mg} / 1$ to $100,000 \mathrm{mg} / 1$, a minor pressure change of about $50 \mathrm{mmH}_{2} \mathrm{O}$ occurred from the vertical interval $\mathrm{H}=500 \mathrm{~mm}$ between the highpressure and the low-pressure side outlets. The pressure change was converted into a 4-20 $\mathrm{mA}$ DC signal and transmitted to a recorder. The recorder saved the data every 20 seconds. In addition, the 4-20 mA DC signal was sent to the dam management office with a telemeter and the measured value was displayed on a monitor in the management office every 10 minutes.
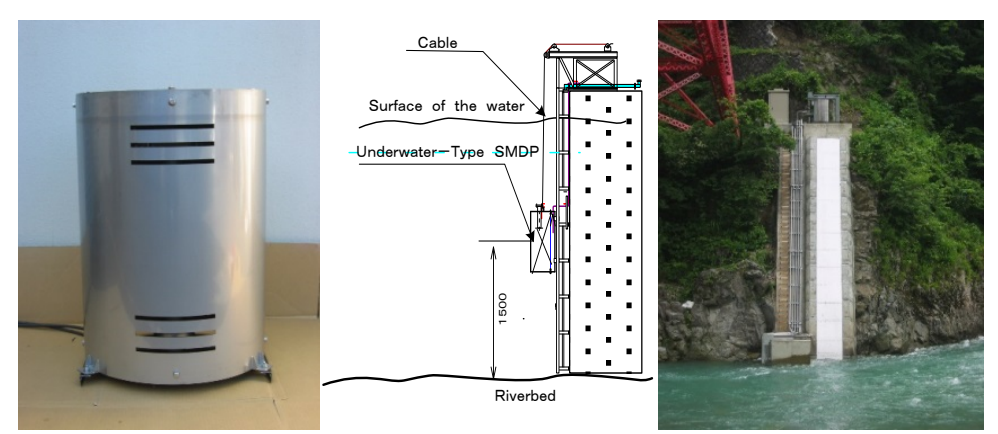

Fig. 2 Underwater SMDP installed downstream of the Unazuki Dam

(Left: SMDP body, Center: Suspension device and installation status, Right: Installation location (approximately 500-m downstream of the Unazuki Dam) 


\section{SS concentration measurement and control results}

\subsection{Results of coordinated sediment flushing in 2016}

Coordinated sediment flushing at the Unazuki Dam and the Dashidaira Dam was carried out from June 25 to 27, 2016. Figs.3 and 4 show flow discharge data and reservoir water level, respectively. At the Unazuki Dam, the peak inflow amount reached $336 \mathrm{~m}^{3} / \mathrm{s}$ at 05:30 on June 25, and the reservoir water level draw down started at 06:40 from $244.6 \mathrm{~m}$. About 8 hours later at 14:32, the sediment-flushing gate was opened when the reservoir water level was $234.6 \mathrm{~m}$. After about 6 hours later, free flow flushing started at 20:45 when the reservoir water level was $220.4 \mathrm{~m}$.

After starting the free flow around 20:45 on June 25, the inflow water flowed through the bottom of the dam in a river-like stream which erodes the sediment accumulated on the bottom of the reservoir. As a result, the SSC in the discharged water becomes the highest. The SSC measurement at this time is important to check the environmental impact on acuatic lifes. Conventionally, at the observation point downstream of the Unazuki Dam, turbidity and SSC measurements were conducted by manual water sampling in one-hour intervals. Generally, since it took several days to obtain the actual SSC after water sampling and the turbidity was indirectly monitored, and the SSC measurements could not be used directly for dam operations.

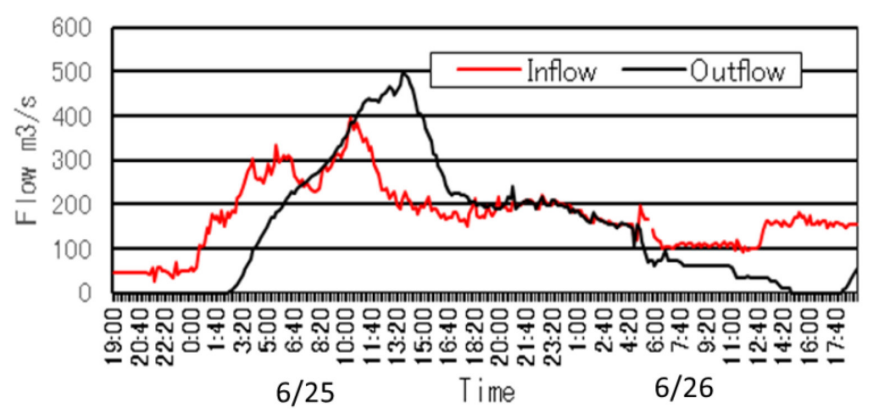

Fig. 3 Flow discharge data of the Unazuki Dam during coordinated sediment flushing in 2016

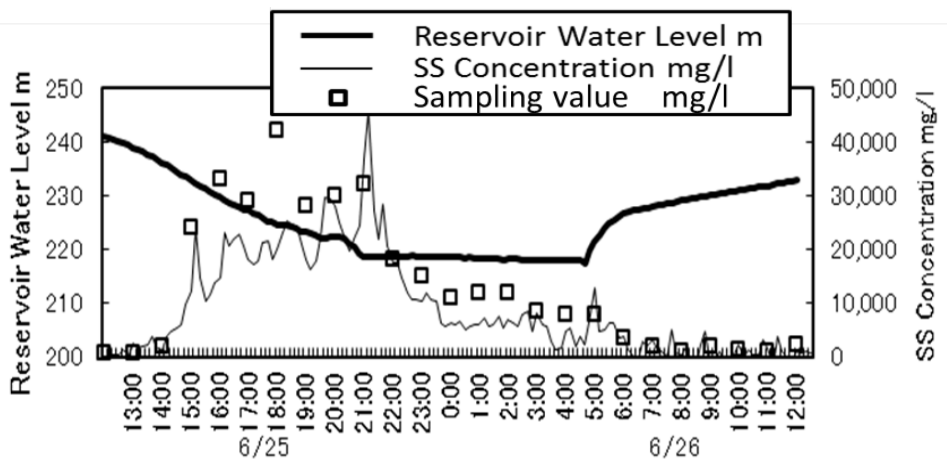

Fig. 4 Reservoir water level and SS concentration during coordinated sediment flushing in 2016

\subsection{Method of gate operation}

The present gate operation for sediment flushing is conducted by setting an upper threshold value for the SSC downstream of the dam. Fig. 5 shows an image of this 
operation. When the SSC gradually increased and approached the upper limit $(30,000 \mathrm{mg} / \mathrm{l})$, the gate will be closed so as to reduce discharge amount by controlling erosion of the riverbed sediment and decreasing the SSC. As the SSC decreased and reached to the lower limit target value $(20,000 \mathrm{mg} / \mathrm{l})$, the gate will be re-opened to increase the discharge to the riverbed sedimentation is more transported. In the actual case at the Unazuki dam, the above operations were repeated several times to draw down the water level while keeping SSC within the target range before shifting into the free flow condition.

Fig. 4 shows the actual record of SSC measured by SMDP downstream of the dam during the coordinated sediment flushing in 2016. The SSC fluctuated between 10,000 mg/l and $30,000 \mathrm{mg} / 1$ due to the gate operations with the above mentioned method. Consequently, draw down operation shifted into free flow without any extreme peak values of SSC. SMDP could measure continuous SSC which is well fitting with the water sampling data even though there was a slight deviation.

Meanwhile, during the coordinated sediment flushing in 2015, the gates were operated without using SMDP to monitor the SSC. The sediment-flushing gate was simply opened to draw down the reservoir water level and shifted into free flow flushing. Fig. 6 shows the changes in the SSC measured by SMDP and the reservoir water level downstream of the dam during coordinated sediment flushing in 2015. Similarly, SMDP measured data is well fitting with the water sampling data.

Fig. 7 compares the SSC during coordinated sediment flushing in 2015 and 2016. The graphs are normalized by setting the time that the flushing gate was opened as time 00:00. This indicates that the SSC of the peak in 2015 rapidly increased to about six times the initial concentration. Meanwhile, in the sediment flushing in 2016, increasing trend of peak SSC values is milder than that in 2015 and it is estimated that gate control by monitoring the SSC downstream of the Unazuki Dam successfully reduced the increase of SSC by limiting about 1.5 times compared to the just before the value. The target sediment flushing amount of the Dashidaira Dam was $290,000 \mathrm{~m}^{3}$ in 2016, which was larger than the 160,000 $\mathrm{m}^{3}$ target in 2015. If the water level was drawn down similar to 2015 without any gate control with careful SSC monitoring, the SSC would have been several times higher than the present one because of larger amount of target sediment volume in 2016.

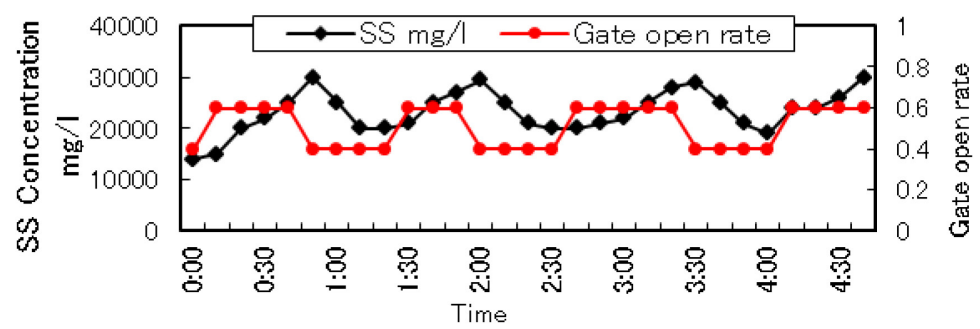

Fig. 5 Examples of gate operations and SS concentration change (Time is an assumed value.)

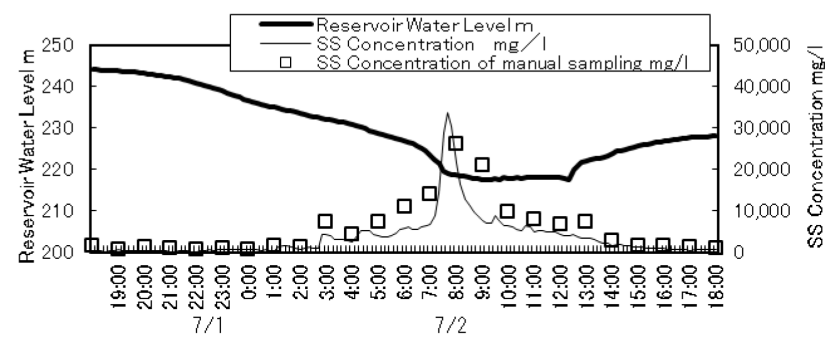

Fig. 6 Reservoir water level and SS concentration during sediment flushing in 2015 


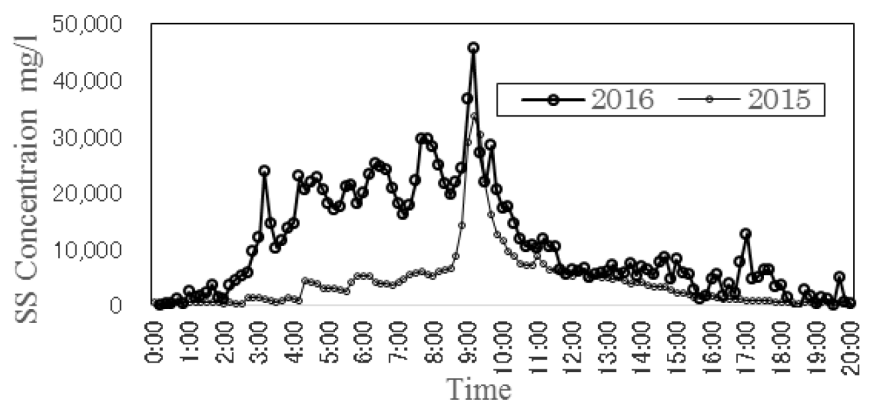

Fig. 7 Comparison of SS concentration changes during coordinated sediment flushing in 2016/2015 (The time is normalized by setting the time that the flushing gate was opened as time 00:00.)

\subsection{Amount of SS discharge}

We can estimate SS discharge volume using SMDP continuous measuring data. The amount of suspended load flowing into the Unazuki Dam varies with the natural conditions over time. Ideally, the suspended load flowing into the dam should be removed. Coordinated sediment flushing is the main opportunity to flush the suspended load flowing into the dam. The annual amount of discharging suspended load depends on the amount of sediment flowing into the dam. Thus, the amount of coordinated sediment flushing will be larger when more suspended load flowed into deposited. Needless to say, the influence on the downstream area must be reduced by controlling the discharging SSC.

It is assumed that the amount of outflow suspended load of a dam $(\mathrm{kg} / \mathrm{s})=\mathrm{SS}$ concentration $(\mathrm{g} / \mathrm{l}) \times$ outflow amount $\left(\mathrm{m}^{3} / \mathrm{s}\right)$. Fig. 8 shows the amount of SS at the time of coordinated sediment flushing in 2016. The amount of discharged SS is spread out instead of concentrating one at one time. In 2016, because of the careful gate operations with precise monitoring the SSC, more large volume of SS was totally discharged without significantly increasing the peak concentration (Fig. 8).

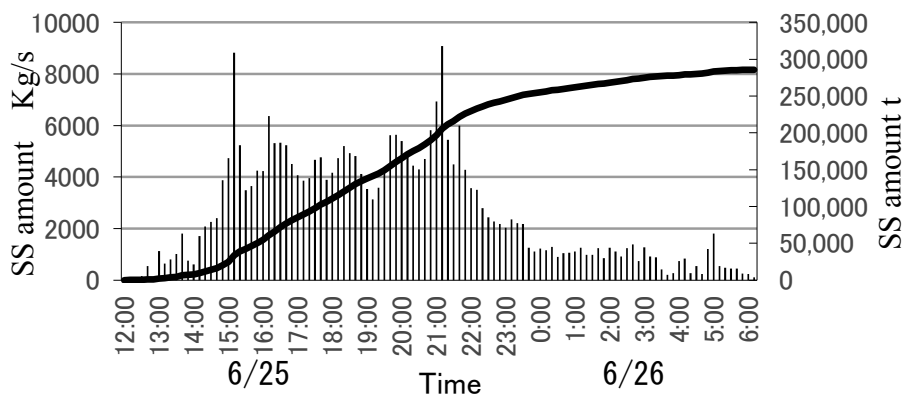

Fig. 8 Estimated SS discharge during coordinated sediment flushing in 2016

\subsection{Comparison of the SSC at the Shimokurobe Bridge and downstream of the dam}

The SSC was also observed on the left bank of the Shimokurobe Bridge to check the water quality just before the river mouth (Fig.1). Since this monitoring point is at a shallow water depth, it was difficult to install the underwater-type SMDP and a water circulation-type SMDP was selected. This type measures the SSC with an SMDP sensor placed in the cylindrical water tank by intaking and circulating the river water with a submergible pump which is suitable to measure medium SSC around 10,000 mg/l. The span of the water 
circulation-type SMDP of the Shimokurobe Bridge was 20,000 mg/l which corresponds to the SSC measurement at the Shimokurobe Bridge. It is considered that suspended load flowing from the Unazuki Dam was mostly discharged into the sea, but some was deposited on the riverbed between the dam and the Shimokurobe Bridge, which is about $20 \mathrm{~km}$ downstream. Fig. 9 compares the concentrations between the Unazuki Dam and the Shimokurobe Bridge in the coordinated sediment flushing in 2016. The peak value of SSC at the Shimokurobe Bridge was reduced to around $20 \%$ of that at downstream of the Unazuki Dam. The suspended load which corresponds to the difference in the SSC between downstream of the Unazuki Dam and the Shimokurobe Bridge was considered to be accumulated on the riverbed. In the Kurobe river, the outflow rinsing discharge with clear water from overflow spillway after reservoir water level recovering is regularly carried out to flush those deposited fine sediment on the riverbed in order to minimize environmental impacts after sediment flushing [9].

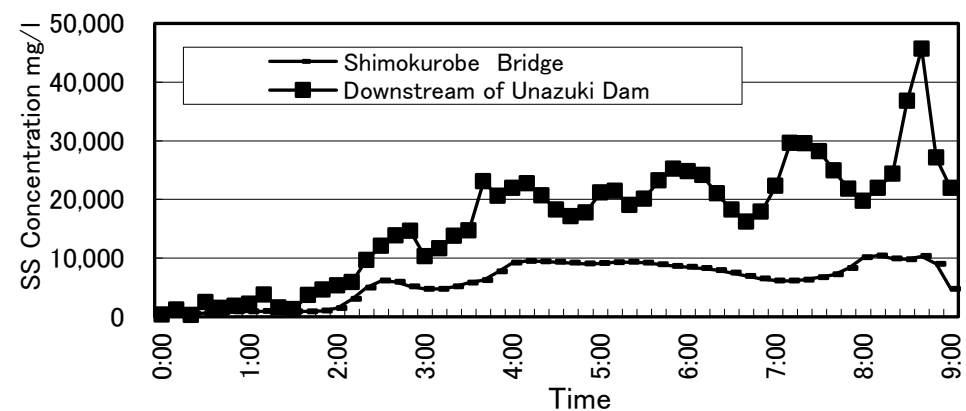

Fig.9 Comparison of SS concentration downstream of the Unazuki Dam and the Shimokurobe Bridge in 2016 (The time is adjusted so as to match the maximum value.)

\section{Conclusion}

This paper shows the results of continuous measurement of high suspended sediment concentration during sediment flushing and sluicing operation of the Unazuki dam in 2015 and 2016 using the Suspended Sediment Concentration Measuring System with Differential Pressure Transmitter (SMDP). Based on the measurement, we have obtained the following conclusions.

- An underwater SMDP downstream of the Unazuki Dam could measure an extreme high SSC up to a peak value of $45,000 \mathrm{mg} / 1$ during coordinated sediment flushing in 2016 which is hardly measured by conventional turbidity meter.

- $\quad$ Adjusting operations of the sediment-flushing gate by monitoring the SSC with SMDP enabled to conduct draw down sediment flushing successfully while keeping the SSC downstream of the Unazuki Dam within the target range in order to minimize environmental impacts.

- In the future, automatic gate operation based on monitoring the SSC during just starting free flow condition can be conducted in order to effectively discharge the suspended load from the reservoir while labor-saving.

- A comparison of the SSC downstream of the Unazuki Dam with the peak value of the SSC of the Shimokurobe Bridge shows the reduction of around $20 \%$ of that which indicates that it is important to clarify the reduction mechanism in the river channel and to conduct the outflow rinsing discharge with clear water to minimize environmental impacts after the sediment flushing. 
Acknowledgments: We express our sincerest gratitude for great cooperation of the Kurobe Office of River, Hokuriku Regional Development Bureau, Ministry of Land, Infrastructure, Transport and Tourism of Japan for the installation of SMDP and provision of the operation and dam hydrologic data, etc.

1. D.G. Wren, B.D. Barkdoll, R.A. Kuhnle and R.W. Derrow, ASCE J. HY 126, 2 (2000)

2. C. Peteil, F. Fruchart et. al., Advances in River Sediment Research - Fukuoka et al. (eds), ISBN 978-1-138-00062-9 (2013)

3. D. Felix, I. Albayrak and R.M. Boes, River Sedimentation, Wieprecht et al. (Eds), ISBN 978-1138-02945-3 (2017)

4. T. Sumi, S. Morita, T. Ochi and H. Komiya, Dam Eng., JSDE, 11, 3 (2001) (in Japanese)

5. T. Sumi, S. Morita, T. Ochi and H. Komiya, Proc. Hydraulic Engineering, JSCE, 46 (2002) (in Japanese)

6. T. Sumi, Biyinbaoligao and S. Morita, Proc. River Technology, JSCE, 10 (2005) (in Japanese)

7. T. Sumi, Biyinbaoligao and S. Morita, J. Hydroscience and Hydraulic Engineering, JSCE, 25, 1 (2007)

8. T. Sumi, S. Morita and H. Komiya, Proc. Hydraulic Engineering, JSCE, 54 (2010) (in Japanese)

9. T. Sumi, S. Nakamura and K. Hayashi, Proc. ICOLD 23rd Congress, Brasilia, Q89-R6 (2009) 